\title{
Workplace Culture and Mental Health Are Interwoven
}

\author{
- \\ STAKEHOLDER RESPONSE \\ John Service, $\mathrm{PHD}$ \\ Canadian Psychological Association \\ $\propto$
}

\begin{abstract}
Workplace health is a serious issue. Illness and injury take a significant toll on employers, employees and the bottom line. Research has focused primarily on the physical health and injury side of the workplace health equation, with much less attention being paid to the mental health factors that affect employers, employees, families, productivity, efficiency, quality, creativity and innovation.
\end{abstract}

WORKPLACE MENTAL HEALTH (behavioural health, mental illness and addictions) is directly related to the workplace environment, home and work balance, employee assistance programs, and the broader public and private healthcare system. We will profit from a greater understanding of the impact of these factors and their interactions with and effects on mental health and resilience.

Many Canadians do not receive any services for their mental health problems. The workplace mental health research agenda can explore the contribution of the

My thanks to Dr. Wendy Josephson, University of Winnipeg and member of the Board of Directors, Canadian Psychological Association, for advice and assistance. 
workplace to improving mental health and resilience for clinical and subclinical populations.

It is important for the CIHR to expand its research activities to include more psychological and social research in order to meet its legislative mandate. This research agenda will help accomplish this important goal, and the results will be generalizable across the work of the institutes.

Kennedy and Bender review important issues relevant to mental health in the workplace. They point out that mental health, mental illness and addictions have a significant impact on the workplace, and they argue correctly that workplace mental health involves a complex interaction of biological, psychological and social factors. This interaction and the relevant drivers within each should be of primary interest in this research initiative. For the purposes of this discussion, mental health includes behavioural health, mental illness and addictions.

The term psychosocial often describes an undifferentiated mass of all things that are not biological or medical. Commonly agreed upon definitions are essential. The biological domain is concerned with, for example, genetics, physiology and pharmacology. The psychological domain focuses on the biological, cognitive, affective, social, cultural and environmental determinants of behaviour (Canadian Psychological Association 1999) and on the behavioural factors affecting health and disease (Dobmeyer et al. 2003). The social domain includes social policy, legislation, regulation, workplace culture, the work-community interface, employee benefits and return-to-work systems.

Kennedy and Bender mention the important contribution of workplace culture to mental health, an issue dealt with in the Vézina paper. These two areas are inextricably interwoven, and the research agenda needs to recognize that fact. The authors also refer to addictions (e.g., to substances or gambling), a serious factor in the workplace that must not become the poor cousin of this research initiative.

Between 30\% to $60 \%$ of primary healthcare visits to family physicians are because of a psychiatric or psychological problem or said problem is a significant contributing factor to the diagnosis (Cummings et al. 1997). Estimates suggest $20 \%$ of the Canadian population will experience a significant mental health problem in their lifetime but fewer than $50 \%$ report receiving treatment (Statistics Canada 2003). It is important to understand the impact of these treated and untreated conditions on workers and the workplace and how the workplace can help to improve employee functioning.

While it is true that the majority of mental health services in Canada are delivered by physicians, this is to some extent an artifact of funding mechanisms and of supply and demand. New interdisciplinary models for primary healthcare and mental healthcare delivery are being developed. These models must take the workers and the workplace into account, and the workplace research agenda must contribute to the development of models that effectively support and interact with the workplace (e.g., examine the interface between primary healthcare and the workplace; study the impact of the increase in the privatization of mental health services; evaluate the role and efficacy of Employee Assistance Programs (EAP) and their interface with work and primary healthcare; and examine back-towork and maintenance programs). 
As Kennedy and Bender state, mental health screening, recognition and treatment in the workplace need to be better understood. This discussion could expand to include conflict resolution, harassment and bullying and their relationship to mental health (LeBlanc and Kelloway 2002). It is also as important to examine the prevalence, course and impact of subclinical conditions, which are widespread and have a considerable effect on the workplace.

Most Canadians work for small and medium-sized organizations that do not have the same work environment, infrastructures, unionization or benefits as large corporations. This sector needs to be included in the research.

Workers bring their external environment to work and vice versa. It is crucial to understand better the impact of nonwork variables on workplace mental health and the interaction between the work and non-work factors that support psychological resilience in workers.

There are structural problems in the collection and sharing of mental health data on epidemiology 1 , utilization and outcomes in Canada (Statistics Canada 2003; Health Canada 2002). These problems are evident in both the public sector (e.g., hospitals, regional health authorities and provincial and territorial governments) and the private sector (e.g., insurers, private providers and EAPs). A study of the currently available data systems in order to make improvements has the potential to significantly strengthen research in this area.

There is excellent research already occurring in the biomedical and pharmaceutical areas within the CIHR. The workplace mental health agenda will profit from this science. The results generalize well. The psychological and social aspects of health, mental health and addictions are less well funded in the 13 CIHR institutes. They are central to the workplace research agenda and provide an important and exciting opportunity. The focus of this research agenda needs to remain on the psychological and social factors.

\section{References}

Canadian Psychological Association. 1999. Strengthening Medicare: The Role of Psychology in the Health of Canadians and in the Development of the Canadian Healthcare System. Ottawa: The Association.

Cummings, N.A., J.L. Cummings and J.N. Johnson. 1997. Behavioural Health in Primary Care: A Guide for Clinical Integration, xxi-xxvii. Madison, WI: Psychosocial Press.

Dobmeyer, A.C., A.B. Rowan, J.R. Etherage and R.J. Wilson. 2003. “Training Psychology Interns in Primary Behavioural Healthcare." Professional Psychology: Research and Practice 34: 586-94.

Health Canada. 2002. A Report on Mental Illness in Canada. Ottawa: Health Canada.

LeBlanc, M.M. and E.K. Kelloway. 2002. "Predictors and Outcomes of Workplace Violence and Aggression." Journal of Applied Psychology 87 (3): 444-53.

Statistics Canada. 2003. Canadian Community Health Survey: Mental Health and Wellbeing. Ottawa: Statistics Canada. 